\title{
Beam Search Algorithm for Fingerprint Reference Point Determination Based on Joint Orientation Features
}

\author{
Shan Juan Xie ${ }^{1}$, Yanshuang Zhang ${ }^{2}$ \\ ${ }^{1}$ Hangzhou Normal University, Institute of Remote Sensing and Earth Science, Hangzhou, 311-121, China \\ ${ }^{2}$ Zhejiang Gongshang University Hangzhou College of Commerce, Hangzhou, 311-121, China
}

\begin{abstract}
In this paper, we propose an efficient beam search algorithm for fingerprint reference point determination with its joint orientation features, which operates in two-step as well as two-path. For the two-path operation, it uses different orientation features at different scales: block-based orientation certainty and pixel-based orientation map. While some fault symptoms are roughly pruned by a block-based orientation feature which is used to ignore some local maxima and select some candidate regions for a reference point region and an accurate path to a reference point is guided by a pixel-based orientation map, their information also is fused for making up one another's weakness. For the two-step operation, in the first step, several candidates of a reference point are determined through beam search algorithm and, in the second step, an accurate reference point is located through the specific concept models. The proposed system has pixel-based precision in determining the position of reference point without complex enhancement, which has been successfully tested through the database FVC2004.
\end{abstract}

Keywords: biometrics; fingerprint; reference point determination; beam search; orientation feature

\section{Introduction}

Automatic fingerprint identification system (AFIS) is one of the most popular and reliable technologies for automatic biometric identification, which is divided roughly into two categories, namely, minutiae level and coarse level. Minutiae level methods take into account the ridge terminations and bifurcations, while coarse level researches the formation of the flow of ridges and valleys. In many fingerprint identification system, a point defined as "the point of the maximum curvature on the convex ridge" [1] is proposed to refer the fingerprint image to be invariant to evident spatial error originated from the scanning process. Moreover, with its prominent curvature characteristic, it is crucial to localize reference points in fingerprint applications such as fingerprint classification and retrieval from large databases, because it can greatly reduce the number of the fingerprints to be compared in the verification step.

Most of the reference point determination algorithms are based on the orientation field estimation techniques, global-based [2], [3], [5-7] and pixel-based orientation methods [4][17]. The typical block-based orientation, Poincare Index (PI) method [6],[7], which is computed by summing changes of orientation around a closed curve of the block, show a poor contrast and loss of the details of ridges, and locate the reference point in the block precision. It performs well except for the arch-type fingerprint or when the orientation change is near $\pi / 2$ or $-\pi / 2$. The pixel-based orientation method [4], although it can label the reference point in the pixel precision, whose performances depend heavily on the quality of the fingerprint image and its continuous orientation map. For example, they can show bad results when fingerprint images have bad quality due to wet or dry acquisition condition or when they are arch-type or loop-type fingerprints. Meanwhile, A.K. Jain et al [8] determine a reference point using the multi-resolution analysis of the differences of sine component integration between any two regions in the orientation filed. And also the complex filtering methods $[9,10]$ detect the maximum curvature based on the ridge orientation field. However these methods still can't perform well for the plain arch type fingerprint.

Because the time consuming nature of PI method and the defects of the pixel-based orientation method, in this paper, two novel orientation features at different scales: block-based orientation certainty and pixel-based orientation map are fused to determine the reference point in pixel-based precision with no requirement of complex enhancement processing. The beam search algorithms firstly try to detect all the potent candidate regions greedily, and then the specific concept models decide the exact location of the reference point. The system which adopts a structure with two steps and paths reduces the computational complexity by that some processes are taken in cascade only at positions selected by the previous filtering and also eliminate many fault symptoms due to some excessive information and its some processes are done in parallel.

\section{Proposed reference point determination system}

To determine the fingerprint reference point, we use a two-step strategy: beam search algorithm is first applied to detect potential candidates with the proposed joint orientation features, and then the specific concept models are proposed to locate the exact reference point within the estimated candidates. The joint orientation features are applied in two paths, which are extracted by two kinds of computational scales and chances of their cross-references for the information fusion. While some fault symptoms are roughly 


\section{International Journal of Science and Research (IJSR) \\ ISSN (Online): 2319-7064}

Index Copernicus Value (2013): 6.14 | Impact Factor (2014): 5.611

pruned by a block-based orientation feature which is used to ignore some local maxima and select some candidate regions for a reference point region and an accurate path to a reference point is guided by a pixel-based orientation map, their information also is fused for making up one another's weakness. Fig. 1 shows its overall block diagram and simplified block diagrams according to its operations. The system which adopts a structure with two steps and paths reduces the computational complexity by that some processes are taken in cascade only at positions selected by the previous filtering and also eliminate many fault symptoms due to some excessive information and its some processes are done in parallel. The proposed system has pixel-based precision in determining the position of reference point through the two-step process of narrowing down and tuning candidates repeatedly.

\section{Joint orientation feature extraction}

The curvature characteristic of the reference point which has the maximum curvature on the convex ridge indicates the change of orientation. Similar as the existing works, our method also characterizes the fingerprint though the orientation patterns. A block-based orientation certainty is used to describe the change of a block curvature of a fingerprint, which is determined by two eigenvalues of its Hessian matrix. And a pixel-based orientation map is used to find intersections emerging from the orientation transition. By calculating the orientation for every pixel, the orientation transitions are estimated to find the point with maximum curvature. Unfortunately, it's not easy to know the accurate pixel orientation, because the point orientation is easily affected by the noise. To overcome the shortcoming of both of two orientation methods, a novel fusion method like the morphologic operation is proposed to join the block orientation information and the pixel orientation information.

\subsection{Block-based orientation certainty feature extraction}

Orientation certainty is considered as an important local analysis method in detecting fingerprint orientation certainty level, which indicates the energy concentration along on the orientation of ridges. Orientation certainty is not only used in the fingerprint, it also be used in detecting edge, ridges, peaks, corners and vertices through the detailed analysis [16]. It can be computed as the ratio of two eigenvalues of the Hessian matrix of the pixel gradient vector.

The intensity gradient $g_{X}, g_{y}$ of each pixel is calculated using Sobel operator with the window size of $3 \times 3$ shown in Eq.1, where $\mathrm{S}$ is the input image.

$$
\mathrm{g}_{x}=\left[\begin{array}{ccc}
-1 & 0 & 1 \\
-2 & 0 & 2 \\
-1 & 0 & 1
\end{array}\right] * \mathrm{~S} \text { and } \mathrm{g}_{y=}\left[\begin{array}{ccc}
-1 & -2 & -1 \\
0 & 0 & 0 \\
1 & 2 & 1
\end{array}\right] * \mathrm{~S}
$$

The Hessian matrix $\mathbf{H}$ of the gradient vector for an $\mathrm{N}$ points image block is given by:

$$
\begin{aligned}
\mathbf{H}=\frac{1}{N} \sum\left(\left[\begin{array}{l}
g_{x} \\
g_{y}
\end{array}\right]\left[\begin{array}{ll}
g_{x} & g_{y}
\end{array}\right]\right)= & {\left[\begin{array}{cc}
G_{x}^{2} & G_{x} G_{y} \\
G_{y} G_{x} & G_{y}^{2}
\end{array}\right]=\left[\begin{array}{ll}
h_{1} & h_{3} \\
h_{3} & h_{2}
\end{array}\right] } \\
& \text { a fingerprint image }
\end{aligned}
$$

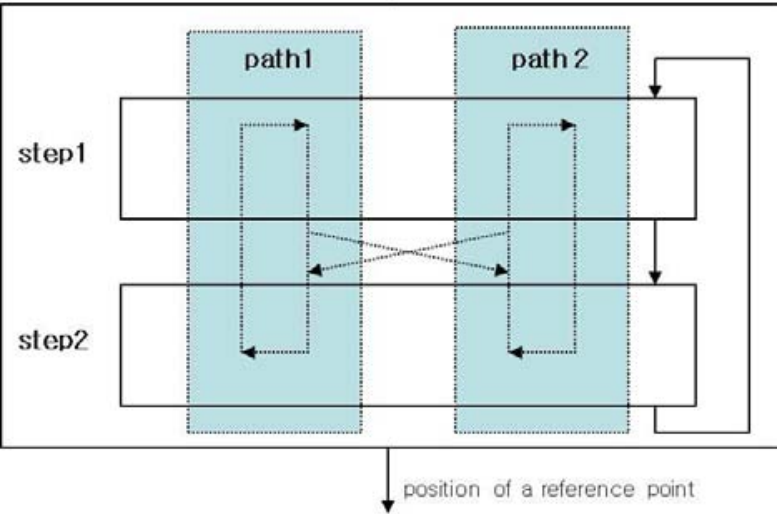

(a) Overall block diagram of the proposed system.

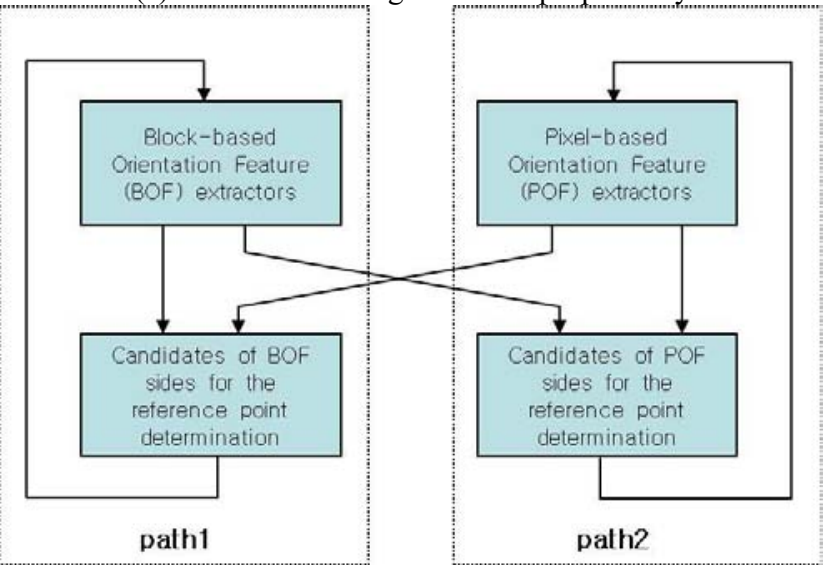

(b) Two-path operation provides the system with the orientation features extracted in two kinds of computational scales and chances of their cross-references for the information fusion.

step1

Beam search for the best potent candidates for reference point determination 


\section{International Journal of Science and Research (IJSR) \\ ISSN (Online): 2319-7064}

Index Copernicus Value (2013): 6.14 | Impact Factor (2014): 5.611

$$
\begin{gathered}
\lambda_{\min }=\frac{\left(h_{1}+h_{2}\right)-\sqrt{\left(h_{1}-h_{2}\right)^{2}+4 h_{3}{ }^{2}}}{2} \\
\lambda_{\max }=\frac{\left(h_{1}+h_{2}\right)+\sqrt{\left(h_{1}-h_{2}\right)^{2}+4 h_{3}{ }^{2}}}{2} \\
\text { Certainty }=1-\frac{\lambda_{\min }}{\lambda_{\max }}
\end{gathered}
$$

The orientation certainty value distributes between the range of $[0,1]$ and indicates the orientation consistency of a block. For a large certainty value, ridges and valleys are very clear with good orientation consistency and, as the certainty value decreases, they change irregularly [15].

To analyze the certainty feature of the fingerprint image, four kinds of regions are separated by their curvature properties, ordinary region, reference point region, edge region and noise region as shown in Fig.2. The ordinary region always shows the stable orientation between ridges, while the reference point region has the abrupt orientation changes between the ridges since the reference point brings the maximum curvature. And the noise regions always cause large curvature due to the noises affect the curvature calculation easily. The edge region also shows large curvature value as the differences exist between the foreground and background. Fig. 3 illustrates the distribution of orientation certainty for the four kinds of regions. For each kind of regions, there are 500 representational sample regions with the size of $32 \times 32$ pixels collected from the database FVC2004_DB1. X-axis is the ratio between the certainty of the tested region, and $\mathrm{Y}$-axis is their frequency value. The four regions show diverse distributions that is helpful to distinguish these four regions. Reference regions are maintained in the range of [0.2 0.6] with the interfere of edge region and noise region. As our purpose of reference point determination, we need to exclude the interferes of noise region and edge region. Rule 1 is concluded for the initialization of candidate reference point region.

Rule 1(Candidate reference point Region Initialization): If the Certainty value of a non-edge block is in an experimental range which is larger than 0.25 times and less than 0.6 times of the average Certainty value of a full image, the block is considered as an candidate reference point region $\mathbf{R}$.

Fig.4 shows an example of block-based orientation certainty map displayed in eight different colors. The orientation certainty values are mapped into the color code from color 1 to color 8 as they decrease. The non-edge blue blocks satisfy the certainty value limitation, and are assigned as the candidate region $\mathbf{R}$.

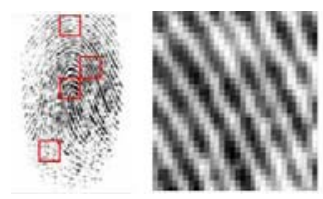

(a) (b)

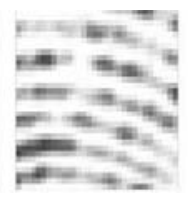

(c)

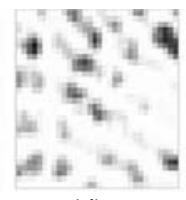

(d)

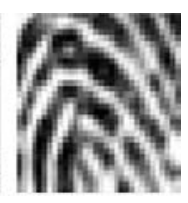

(e)
Figure 2: Four typical sample regions of a fingerprint image, (a) sample testing image, (b) ordinary region, (c) edge region, (d) noisy region, and (e) reference point region.

\subsection{Pixel-based orientation map extraction}

Generally speaking, a fingerprint can be described as a set of ridges flowing concentrically, whose orientations change gradually around the reference point. One will notice that the reference point is not only the maximum curvature on the convex ridge, but also the orientation translation intersections of the image. To find intersections emerging from the orientation transition, the pixel orientation map is firstly

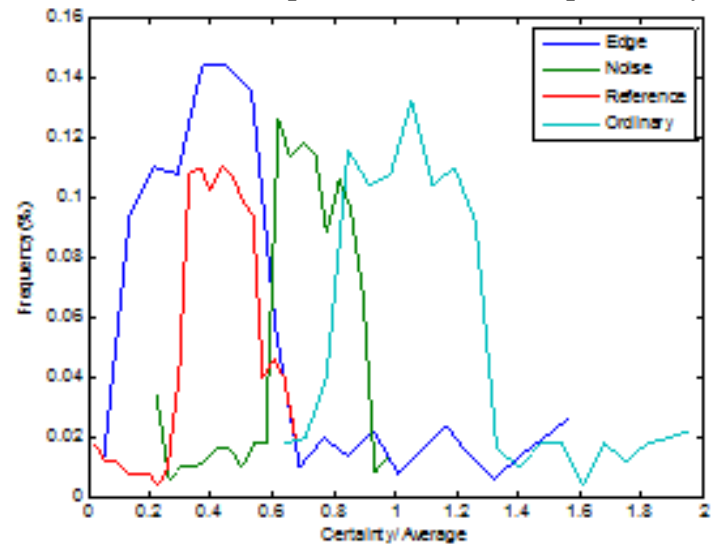

Figure 3: Orientation certainty distributions of the four specific regions.
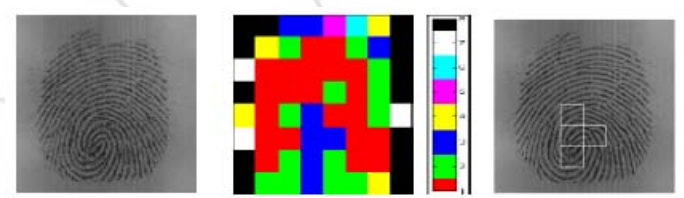

Figure 4: An example of fingerprint orientation certainty map. (a) Tested fingerprint image. (b) Orientation certainty map. (c) Detected Maximum Curvature Regions.

by using arcus tangent [4] for each given pixel $P$ at $(i, j) \in S$.

$$
\alpha_{i j}=\frac{1}{2} \operatorname{atan}\left(\frac{Y_{\mathrm{ij}}}{X_{\mathrm{ij}}}\right)
$$

Where,

$$
X_{i j}=\frac{G_{y}{ }^{2}(\mathrm{i}, \mathrm{j})-G_{x}{ }^{2}(\mathrm{i}, \mathrm{j})}{G_{y}{ }^{2}(\mathrm{i}, \mathrm{j})+G_{x}{ }^{2}(\mathrm{i}, \mathrm{j})}, \quad Y_{i j}=\frac{2 G_{x}(\mathrm{i}, \mathrm{j}) G_{y}(\mathrm{i}, \mathrm{j})}{G_{x}{ }^{2}(\mathrm{i}, \mathrm{j})+G_{y}{ }^{2}(\mathrm{i}, \mathrm{j})}
$$

Usually, imposter intersections are easily interfered, since the ridges looks disconnected with some blur when no complex enhancement processing is applied. Much noise in the pixel orientation map shown in Fig.5(c), thus many enhancement methods are needed. For our algorithm, we use the original image without any enhancement processing, while normalize the pixel orientation into $\mathrm{N}$ levels.

$$
\Phi_{i j}=\left|\frac{\alpha_{i j}}{(\pi / \mathrm{N})}\right|
$$

The normalized orientation map divide the orientation map into $\mathrm{N}$ regions. Between each distinct regions, the segmentation edges are called as transition line. From experimental results, four-level normalization is the most effective to find intersection points between levels for the detection of reference points. The intersection points are hard to represent the reference points when two-level normalization is implemented. On the other hand, when its level is five or 


\section{International Journal of Science and Research (IJSR) \\ ISSN (Online): 2319-7064}

Index Copernicus Value (2013): 6.14 | Impact Factor (2014): 5.611

larger, much more noise pixels are considered as the singular points.

After obtaining the four-level orientation map, morphological closing operations are applied to fill the small holes caused by the noises. Fig. 5(b) shows the smoothed segmented orientation map. From the smoothed orientation map, intersection points can be located by summing the amounts of orientation transition in its $3 \times 3$ neighborhood. It should keep in mind that intersection emerging from the orientation transition that orientation transits from one level to another through the intersection. Thus, pixels that have $\mathrm{N}$ times of transition, or including all the orientation levels in its neighborhood are the intersection points. While for a normal pixel, there is no translation included since all its neighborhood pixels belong to the same orientation level. The transition lines are illustrated in Fig.6, where the triangle regions represent the detected intersection points. Rule 2 used to find candidate intersection points (I) can be summarized as:

Rule 2 (Intersections initialization): If there are four different orientations included in the $3 \times 3$ neighborhoods of a pixel, then the pixel is considered as the intersection point.

These procedures may be illustrated using an algorithm as follows:

1)Calculate the orientation for each pixel, and build the orientation map in the range of $[0, \pi]$.

2) Normalize the orientation map into $\mathrm{N}$ levels.

3)Fill the small noise region in the orientation map by using the morphological closing operation.

4)Detect the transition line and candidate intersections by summing the transition times.

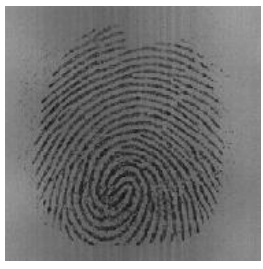

(a)

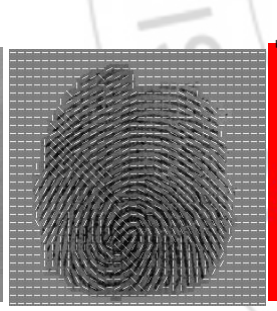

(b)

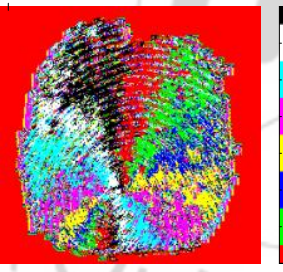

(c)
Figure 5: An example of a fingerprint image segmentation for filtering out its background. (a) An original image. (b) Block-based orientation map. (c) Pixel-based orientation map

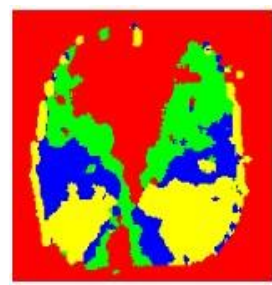

(a)

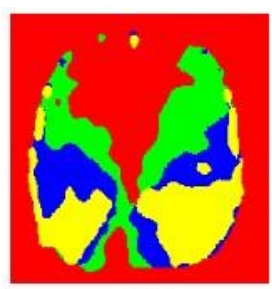

(b)

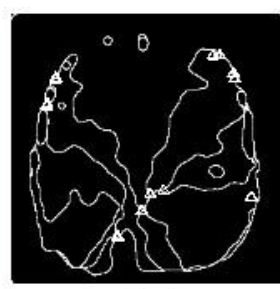

(c)
Figure 6: An example of segmented orientation map. (a) Four-level segmented orientation map. (b) Its smoothed map

(c) The orientation transition lines and the candidate intersections

\subsection{Orientation feature fusion}

The block-based orientation certainty feature concludes the general curvature properties of image, while it easily loss the detail of ridges that resulting the reference point located in the block precision rather than the pixel precision. Moreover, when a fingerprint image has a poor contrast or a big area of noise, a region containing a real reference point may be missed as one of the candidate by the Rule 1 . However, when only pixel-based orientation feature is used, there may be so many fault symptoms due to noises, so it may require additional complex processing, and also the intersection point detection for the all image is time consuming. To solve this problem, we join the orientation certainty and intersections as a new feature, we define the candidate region $\mathbf{R}$ and intersections $\mathbf{I}$ are correlative when $(\boldsymbol{R} \cap \boldsymbol{I}) \notin \varnothing$.

To find the exact position of reference point, we scale down the candidate region with pyramid process. $\mathrm{N}$ numbers of regions with the size of $r_{\mathbf{m}} \times r_{\mathbf{m}}$ pixels construct the m-scale candidate regions, where $\mathbf{R}_{\mathrm{m}}=\left\{\mathbf{R}_{\mathrm{m} 1}, \mathbf{R}_{\mathrm{m} 2}, \mathbf{R}_{\mathrm{mn}}\right\}$. And the new candidate region $\mathbf{R}$ in a lower scale level is denoted as $\mathbf{R}_{\mathbf{m + 1}}$, whose region size is quarter of $\mathbf{R}_{\mathrm{ms}}$.

In the candidate region initialization process, we narrow the orientation certainty range to avoid the interferes of edge and noise region. When a fingerprint image has a poor contrast, arch type or containing a big noisy area, a region containing a real reference point may be missed. To expand the possible candidate region, we joint the intersections point as a guide using the joint dilation process. A new candidate region $\mathbf{R}_{\text {Ims }}$ will be dilated and the correlative intersection $\mathbf{I}_{\mathbf{m s}}$ is taken as the dilation center of the structuring element $\mathbf{E}$.

$$
\mathbf{R}_{\text {Ims }}=\text { dilate }\left(\mathbf{I}_{\mathbf{m s}}, \mathbf{E}\right)
$$

Where $\mathbf{I}_{\mathbf{m s}}=\mathbf{R}_{\mathbf{m}} \cap \mathbf{I}_{\mathbf{m}}$, and $\mathbf{E}$ is the $\mathrm{r}_{\mathrm{m}} \times \mathrm{r}_{\mathrm{m}}$ square region.

Tens or hundreds of intersections are set as candidate intersections which contain all of the possible orientations in a $3 \times 3$ neighborhood. And many of them are pseudo due to the small area noise such as salt noise. The scale pyramid process scale the candidate region into a lower resolution domain where less noise can survive, and only the intersections correlative to the candidate region will be saved as the best hypothesis of reference point. The joint processing for intersections shrinking is named as joint erosion operation defined in Eq.9.

$$
\mathbf{I}_{\mathrm{m}+\mathbf{1}}=\left(\mathbf{R}_{\mathrm{m}} \cap \mathbf{I}_{\mathrm{m}}\right)
$$

when $\left\{\left(\mathbf{R}_{\mathbf{m}} \cap \mathbf{I}_{\mathbf{m}}\right) \notin \varnothing\right\}$, otherwise, $\mathbf{I}_{\mathbf{m}+\mathbf{1}}=\mathbf{I}_{\mathbf{m}}$.

Fig.7 shows an example for the arch-type images. We can see the intersection points cannot be correctly detected by the intersection map. While with the help of orientation certainty, the real intersection is contained in the candidate regions. In Fig. 8, false candidate regions are brought in when the image is thepartially poor. The joint orientation features overcome the noise interferences and detect the real intersection together.

\subsection{Beam Search algorithm for candidates}

With any greedy search, there is a danger that suboptimal choice will be made at any step. To reduce this risk, we can 


\section{International Journal of Science and Research (IJSR) \\ ISSN (Online): 2319-7064 \\ Index Copernicus Value (2013): 6.14 | Impact Factor (2014): 5.611}

extend the algorithm to perform a beam search; that is, a search in which the algorithm maintains a list of the $\mathrm{K}$ best candidates at each step, rather than a single best candidate. On each search step, specializations are generated for each of these $\mathrm{K}$ best candidates, and the resulting set is again reduced to the $\mathrm{k}$ most promising members. Beam search keeps track of the most promising alternatives to the current top-rated hypothesis, so that all of their successors can be considered at each search step.

Throughout the search procedure, partial hypotheses are maintained to select the candidates. The candidate hypothesis of reference point is firstly initialized as the maximum curvature region $\mathbf{R}$ and the candidate intersections I. Scale pyramid process generate the more specify candidate region $\mathbf{R}_{\mathbf{m}}$ with M levels of resolution. The number of resolution is depended on the size of image and the initialized candidate region. $\mathrm{K}$ candidate regions with the minimum orientation certainty will be selected as the new candidate regions $\mathbf{R}_{\mathbf{m + 1}}$, as well as the dilated region $\mathbf{R}_{\text {Ims }}$ by the correlative candidate intersections $\mathbf{I}_{\mathbf{m s}}$ using the joint dilation operation. Meanwhile, an efficient intersections update algorithm is proposed by restriction the possible intersections inside the candidate region.

The candidate intersections will keep in the next scale level until the correlative intersections are found and updated by the joint erosion process. The best hypotheses are determined through the orientation certainty variance test and orientation sequence checking. A detail description of the beam search algorithm is given in the Table.1.

Table 1: Beam search algorithm for candidate reference point determination

- Initialize Best-hypothesis to the most general hypothesis using Rule 1(Candidate Regions initialization), and Rule 2 (Intersection points initialization).

- Initialize Candidate-hypothesis: $\boldsymbol{C H}=\{(\boldsymbol{R}, \boldsymbol{I})\}$ to the set $\{$ Best-hypothesis $\}$

For $\mathrm{m}=1,2, . . \mathrm{M}-1$, where $\mathrm{M}$ is determined by the image size and resolutions,

While Candidate-hypothesis $\left\{\left(\boldsymbol{R}_{\boldsymbol{m}} \cup \boldsymbol{I}_{\boldsymbol{m}}\right) \notin \varnothing\right\}$, Do

1. Generate the next more specific Candidate-hypothesis

a. Subdivide each candidate region in $\boldsymbol{R}_{\boldsymbol{m}}$.

Find K sub-regions $\boldsymbol{R}_{\boldsymbol{m}+1}$ with the minimum orientation certainty.

Add new candidate region with intersections dilate operation: $\mathbf{R}_{\mathbf{I m s}}=\operatorname{dilate}\left(\mathbf{I}_{\mathbf{m s}}, \mathbf{E}\right)$

b. New candidate intersection with erosion operation: $\mathbf{I}_{\mathbf{m}+\mathbf{1}}=\left(\mathbf{R}_{\mathbf{m}} \cap \mathbf{I}_{\mathbf{m}}\right)$, when

$$
\left\{\left(\mathbf{R}_{\mathbf{m}} \cap \mathbf{I}_{\mathbf{m}}\right) \notin \varnothing\right\} \text {, otherwise, } \mathbf{I}_{\mathbf{m}+\mathbf{1}}=\mathbf{I}_{\mathbf{m}} \text {. }
$$

2. Update New Candidate-hypotheses: $\boldsymbol{C H}=\left\{\left(\boldsymbol{R}_{\boldsymbol{m}+1}, \boldsymbol{I}_{\boldsymbol{m}+1}\right)\right\}$

3. Update Best-hypotheses

For all hypotheses in New-candidate-hypothesis do

a. Find the candidate regions whose sub-regions satisfy the rule: the variance of orientation certainty of each four sub-regions should follow the range $[0.3,0.8]$.

Fnd

b. Check orientation sequence of candidate intersections

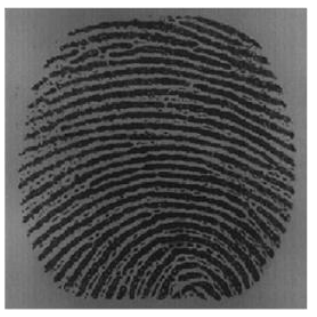

(a1)

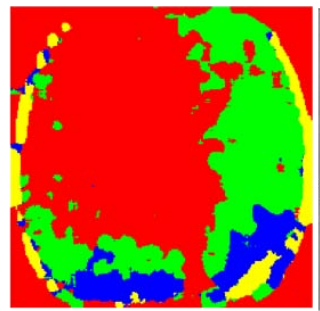

(b1)

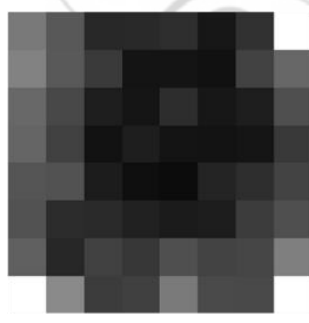

(a2)

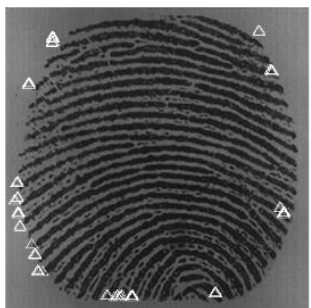

(b2)

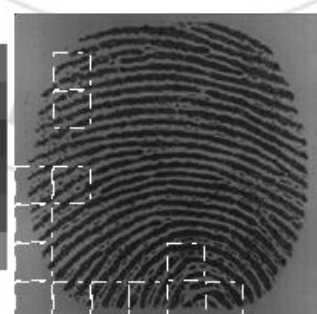

(a3)

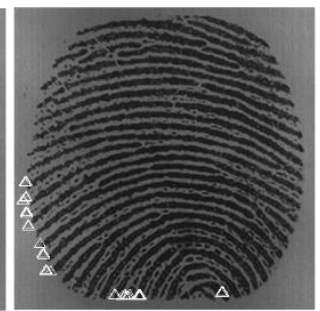

(b3)

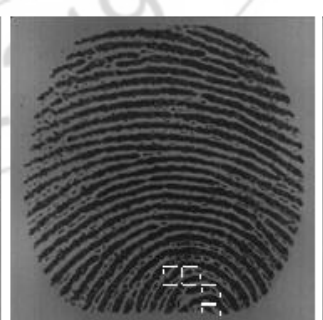

(a4)

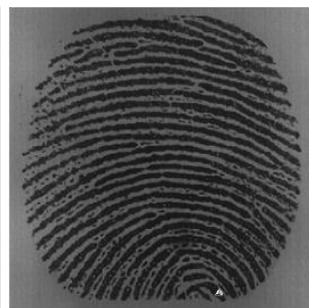

(b4)

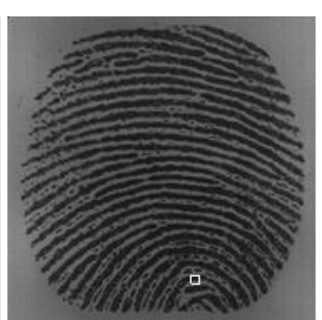

(a5)

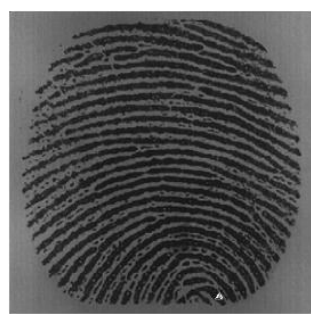

(b5)

Figure 7: Our proposed method for arch type image : (a1) the arch-type image,(a2) the orientation certainty map,(a3)1st candidate region R1,(a4) 2nd candidate region R2,(a5)3rd candidate region R3,(b1) pixel orientation map, (b2) 1st candidate intersections I1, (b3)2nd candidate intersections I2, (b4) 3rd candidate intersections I3, (b5) final reference point.. 


\section{International Journal of Science and Research (IJSR) \\ ISSN (Online): 2319-7064}

Index Copernicus Value (2013): 6.14 $\mid$ Impact Factor (2014): 5.611

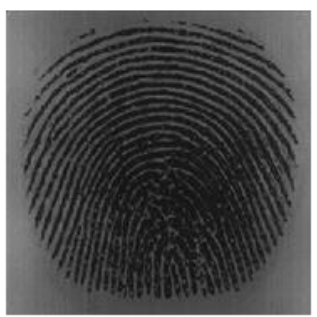

(a1)

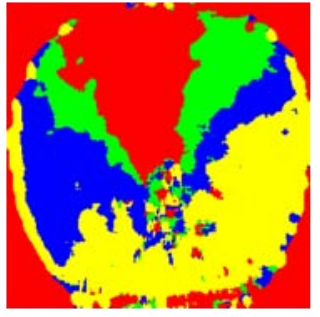

(b1)

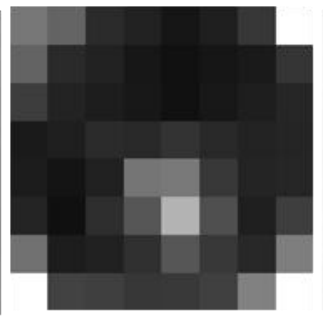

(a2)

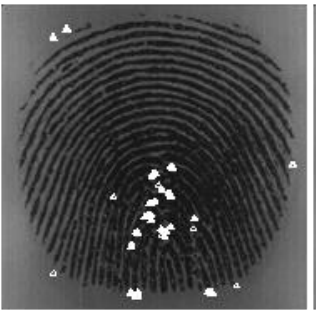

(b2)

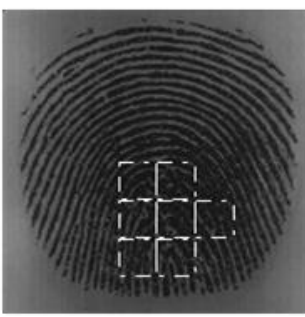

(a3)

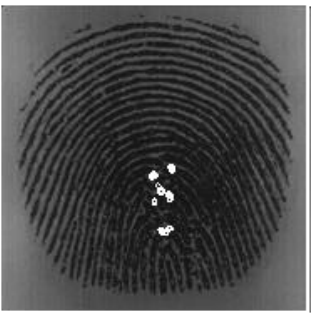

(b3)

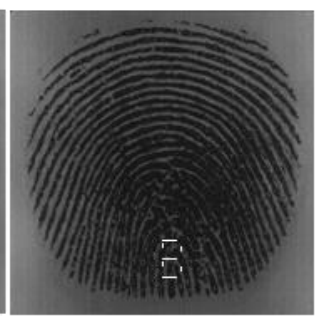

(a4)

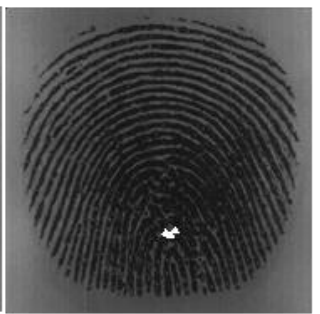

(b4)

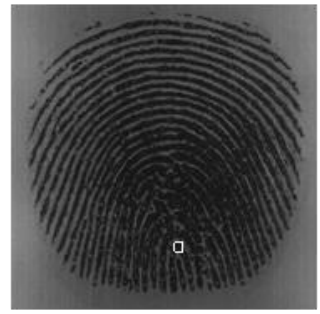

(a5)

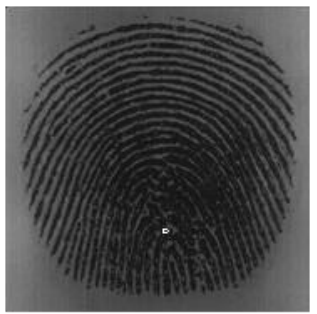

(b5)

Figure 8: Our proposed method for noisy images: (a1) the arch-type image,(a2) the orientation certainty map,(a3)1st candidate region R1,(a4) 2nd candidate region R2,(a5)3rd candidate region R3,(b1) pixel orientation map, (b2) 1st candidate intersections I1, b3)2nd candidate intersections I2, (b4) 3rd candidate intersections I3, (b5) final reference point..

\subsection{Reference point location}

\subsubsection{Orientation sequences tuning}

A reference point is defined as a location where the ridge curvature reaches a local maximum. For the arch type fingerprint, the point of the maximum curvature can't be detected by the intersections. This is also one reason why we fuse the block-based orientation certainty information with the pixel-based orientation information. For the non-arch type, the reference point is always the core singular point. The core singular point is the focus of a set of concentric converging ridges flow, and a delta is the merging point of three cores placed together. To distinguish core singular points with delta singular points and also to distinguish the singular point with noise point, the orientation sequences of intersections are tested. Fig. 9 shows the illustration of these three kinds of points. Fig.10 shows the orientation sequences of pseudo intersections and real intersections.

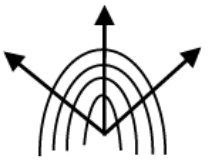

(a)

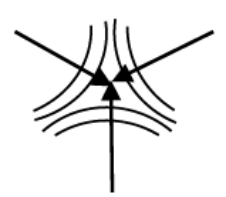

(b)

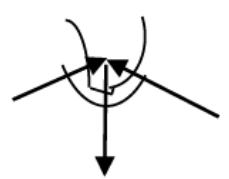

(c)
Figure 9: The orientation illustration of three kinds of points. (a): Core singular point, (b): Delta singular point, (c): Noise point with large curvature.

The orientation sequence is the orientation flow in a neighborhood of the candidate intersections. Let the intersection starts from the top-left pixel in a clockwise manner back to the starting pixel without repetition in the middle.
Rule 4: If its orientation sequence satisfies one of the following cases $U$, the candidate intersections will be saved, otherwise it will be deleted.

$$
\mathrm{U}=\{(4-3-2-1-4),(1-4-3-2-1),(2-1-4-3-2),(3-2-1-4-3)\}
$$

\subsubsection{Reference point location correlation}

After the beam search, at least one best hypothesis array can be detected. The array can be concluded into two cases:

Case 1: $\left(\mathrm{R}_{\mathrm{M}} \cap \mathrm{I}_{\mathrm{M}}\right) \notin \emptyset$

If the candidate intersections $I_{M}$ is correlative with the candidate region $R_{M}$, the center of the intersections satisfying the orientation sequences is located as the exact reference point.

Case 2: $\left(\mathrm{R}_{\mathrm{M}} \cap \mathrm{I}_{\mathrm{M}}\right) \in \emptyset$

If the candidate intersections $I_{M}$ and the candidate region $R_{M}$ are not correlative, the reference point is decided as the pixel in the $R_{M}$ which is nearest to the center of candidate intersections $I_{M}$. Fig. 11 shows this special case when $R_{M}$ and $I_{M}$ are not correlative. The marked pixel which is the nearest pixel to $I_{M}$, shows a better performance compared with the literature method of setting the center of $R_{M}$ as the reference point.

After the beam search, at least one best hypothesis array can be detected. The array can be concluded into two cases:

Case 1: $\left(\mathrm{R}_{\mathrm{M}} \cap \mathrm{I}_{\mathrm{M}}\right) \notin \emptyset$

If the candidate intersections $\mathrm{I}_{\mathrm{M}}$ is correlative with the candidate region $R_{M}$, the center of the intersections satisfying the orientation sequences is located as the exact reference point.

Case 2: $\left(\mathrm{R}_{\mathrm{M}} \cap \mathrm{I}_{\mathrm{M}}\right) \in \emptyset$

If the candidate intersections $I_{M}$ and the candidate region $R_{M}$ are not correlative, the reference point is decided as the pixel 


\section{International Journal of Science and Research (IJSR) \\ ISSN (Online): 2319-7064}

Index Copernicus Value (2013): 6.14 | Impact Factor (2014): 5.611

in the $R_{M}$ which is nearest to the center of candidate intersections $I_{M}$. Fig.11 shows this special case when $R_{M}$ and $I_{M}$ are not correlative. The marked pixel which is the nearest pixel to $I_{M}$, shows a better performance compared with the literature method of setting the center of $R_{M}$ as the reference point.

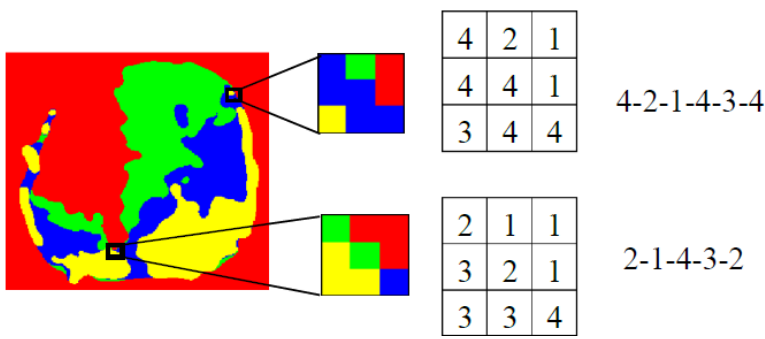

Figure 10: Orientation sequences of pseudo intersections and real intersections

(a)

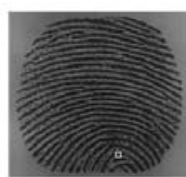

(b)

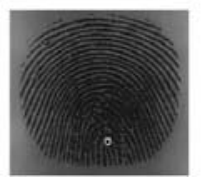

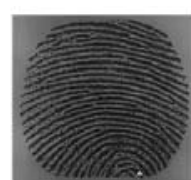

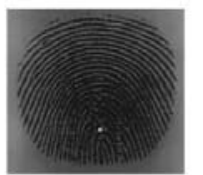

Figure 11: Example for the special case: the candidate intersections IM and the candidate region RM are not correlative.
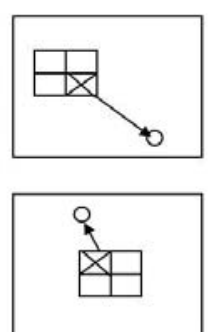

\section{Experimental Results}

\subsection{Database}

The proposed algorithm has been tested on the FVC2004 DB1, DB2, DB3 and DB4. Each database consists of 800 fingerprint images from 100 fingers (with 8 images from each finger). DB1 and DB2 were captured by optical sensor, DB3 was capacitive sensor and DB4 was synthetic fingerprint generation. The image size is adjusted to $256 \times 256$ pixels and the resolution is 500 dpi.

In the databases, there're many poor-quality fingerprints such as partial images with reference points left outside and images with heavy noise like scars, ridge breaks too wet or dry fingerprints, and so forth. Qualities of the four databases are estimated by optimized orientation certainty method [15], the results are shown in Fig.12. The DB1, DB2 databases have the better quality than DB3 and DB4 database.

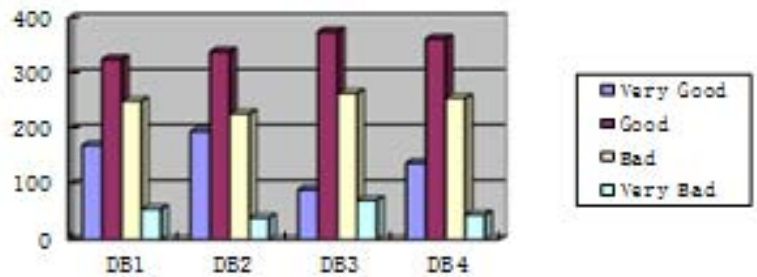

Figure 12: Quality distribution of the FVC2004 database.

\subsection{Accuracy and Complexity}

The desired position and orientation of the reference point in each fingerprint are not detected previously by experts. To evaluate the performance of our proposed algorithm quantitatively, we manually assigned the desired position of the reference point and the desired reference orientation of each fingerprint. The Euclidean distance between the manually-located reference point and the detected reference point is computed.

Fig.13 shows the position accuracy of the reference points of FVC2004 DB1, DB2, DB3, and DB4. The proposed method is compared with complex filter method proposed by A.K. Jain et al. [8], Orientation Certainty based method and the intersections detection method based on pixel orientation map. From the result, we can see the proposed method can determine the reference point location more precisely. For FVC2004 DB1, DB2, DB3, and DB4, The proposed method shows that the accuracy with smaller than 8 pixels distance error is $94.25 \%, 92.13 \%, 92.5 \%$, and $93.25 \%$, respectively. And its accuracy within 16 pixels distance error can reach to $98.63 \%, 98.25 \%, 97.63 \%$, and $97.63 \%$. The proposed method has little effected by fingerprint quality. Table 2 shows the further analysis that our proposed method can concentrate the errors between $[0,8]$ pixels. In the other words, our proposed method is a pixel precision method, and also adapt for the arch type images with the accuracy of $94 \%$. Besides the advantage of higher accuracy, the proposed method is not time consuming due to the dynamic processing are processed parallelly.

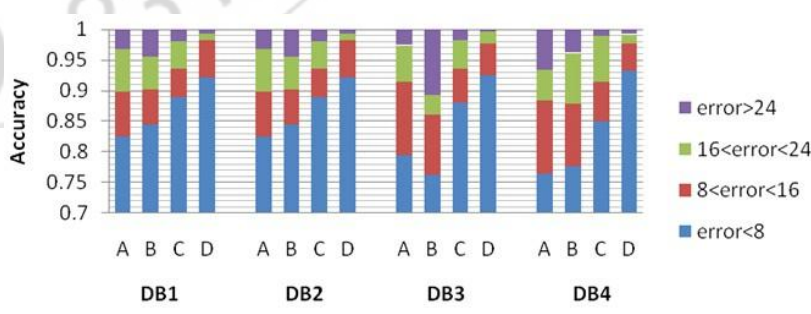

Figure 13: Comparisons of reference point determination accuracy for FVC2004 DB1, DB2, DB3, DB4 by four methods. (A, B, C, and D represent the block-based method, pixel-based method, complex filter method and the proposed method respectively).

Table 2: Results of the proposed reference point determination algorithm on FVC database.

\begin{tabular}{|c|c|c|c|c|}
\hline Methods & block-based & Pixel-based & Complex filter & Proposed \\
\hline Average Distance Error & 16.53 & 8.60 & 12.47 & 5.79 \\
\hline Accuracy for arch type (\%) & $62 \%$ & $81 \%$ & $88 \%$ & $94 \%$ \\
\hline Time(/s) & 0.94 & 1.26 & 2.0 & 0.68 \\
\hline
\end{tabular}




\section{International Journal of Science and Research (IJSR) \\ ISSN (Online): 2319-7064}

Index Copernicus Value (2013): 6.14 | Impact Factor (2014): 5.611

Table 3: Reference point determination accuracy for FVC2004 database classified into four groups according to their image quality

\begin{tabular}{|c|c|c|c|c|}
\hline Distance Error & Very Good & Good & Bad & Very Bad \\
\hline$[0,8]$ & $94.10 \%$ & $93.15 \%$ & $92.47 \%$ & $91.90 \%$ \\
\hline$[9,16]$ & $5.90 \%$ & $5.5 \%$ & $4.72 \%$ & $0.095 \%$ \\
\hline$[17,24]$ & $0.38 \%$ & $1.36 \%$ & $2.31 \%$ & $1.43 \%$ \\
\hline$>24$ & $0 \%$ & $0 \%$ & $0.06 \%$ & $5.71 \%$ \\
\hline
\end{tabular}

\section{Conclusion}

The proposed reference point determination system operates in parallel two-path to fuse block-based orientation certainty and pixel-based orientation map at different scales. The beam search algorithm detects the best reference point candidates through looking up the joint orientation feature, and an accurate reference point is selected from the candidates through the tuning process. The proposed system reduces the computational complexity by that some processes are taken in cascade only at positions selected by the previous filtering and also eliminate many fault symptoms due to some excessive information and some processes are done in parallel. Our proposed method can concentrate the errors between $[0,8]$ pixels, that is smaller error than block orientation based methods and complex filter method. Besides, compared with the traditional intersection determination method, the proposed method also avoids complex enhancement for fingerprint image and the continuous orientation map.

\section{Acknowledgments}

This material is based upon work funded by Zhejiang Provincial Natural Science Foundation of China under Grant No.Q14F010024, the Scientific Research Foundation of Hangzhou Normal University., and the Innovation and Entrepreneurship Foundation for the Returned Overseas Chinese Scholars, Hangzhou.

\section{References}

[1] J. C. Yang, D. S. Park, "A Fingerprint Verification Algorithm Using Tessellated Invariant Moment Features", Neurocomputing, Vol. 71(10-12) pp. 1939-1946, 2008.

[2] D.J. Li, X.S. Yue, Q.X. Wu, W.X. Kang, "CPGF: Core Point Detection from Global Feature for Fingerprint", Biometric Recognition, Vol. 9428, pp 224-232,2015

[3] M. Liu, X.D. Jiang, A. Kot, "Fingerprint reference-point detection”, EURASIPJ. Appl. Signal Process. Vol. 2005 (4), pp:498-509, 2005.

[4] H.K. Lam, Z. Hou, W.Y. Yau, T.P. Chen, “A Systematic Topological Method for Fingerprint Singular Point Detection", ICARCV 2008, pp: 967-972, 2008

[5] B.M. Merthe, N.N. Murthy, S. Kapoor, B. Chatterjee, "Segmentation of fingerprint images using the orientational image". Pattern Recognit. Vol. 20, pp: 429-435, 1987.

[6] K. Karu and A. K. Jain, "Fingerprint classification", Pattern Recognition, vol. 29(3), pp:389-404, 1996.

[7] Q. Zhang, H. Yan, "Fingerprint classification based on extraction and analysis of singularities and pseudo ridges, Pattern Recognition ,vol.37 ,pp:2233-2243,2004

[8] A.K. Jain, S. Prabhakar, L. Hong, S. Pankanti, "Filterbank-based fingerprint matching, IEEE Trans. Image Process. Vol. 9 (5), pp:846-859,2000

[9] M. Liu, X.D. Jiang, A. Kot, "Fingerprint retrieval by complex filter responses", International Conference on Pattern Recognition (ICPR),pp:1042-1045, 2006.

[10] Mohammed S. Khalil, "Reference point detection for camera-based fingerprint image based on wavelet transformation", BIOMEDICAL ENGINEERING ONLINE, vol 14, pp:40, 2015

[11] J. C. Yang, D. S. Park, S. Yoon, "Reference Point Determination in Enhanced Fingerprint Image", ISCID '08, Vol: 2, pp: 161-164,2008

[12] C. Huang, L. Liu, and D.C. Douglas Hung, "Fingerprint analysis and singular point detection", Pattern Recognition Letters, vol. 28(15), pp.1937-1945, 2007.

[13] V.S. Srinivasan, N.N. Murthy, "Detection of singular points in fingerprint images". Pattern Recognit.vol. 25, pp:139-153. 1992.

[14] N. Ostu, " A threshold selection method from gray-level histograms", IEEE Trans. Syst., Man, Bybern.,vol.9, pp.62-66, 1979

[15] S.J. Xie, J.C. Yang, S.Yoon, D.S. Park, “An Optimal Orientation Certainty Level Approach for Fingerprint Quality Estimation,” Intelligent Information Technology Application, Vol. 3, pp.722-726,2008

[16] S. Ando, "Image field categorization and edge/corner detection from gradient covariance", Transactions on Pattern Analysis and Machine Intelligence, IEEE, pp.179-190, 2000

[17] R. Doroz, K.Wrobel, M. Palys, "Detecting the Reference Point in Fingerprint Images with the Use of the High Curvature Points". Lecture Notes in Computer Science, 2015, 9012:82-91

\section{Author Profile}

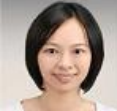

Shan Juan Xie is a lecturer in Hangzhou Normal University, who received her M.S. and Ph.D. degree from Chonbuk National University, Republic of Korea. Her areas of interest are biometrics, pattern recognition, image processing, and machine learning, in which she has authored almost 40 papers in related international journals and conferences.

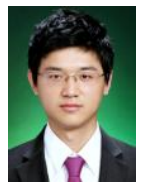

Yanshuang Zhang is a lecturer in Zhejiang Gongshang University Hangzhou College of Commerce, who received his M.S. and Ph.D. degree from Chonbuk National University, Republic of Korea. His areas of interest are Artificial intelligence and Decision making 